\title{
Scattering theory formalism for the magneto-optics of nanocorrugated ferromagnet surfaces
}

\author{
A. Bengoechea, G. Armelles, * J. L. Costa-Krämer, and J. Anguita \\ Instituto de Microelectrónica de Madrid, IMM (CNM-CSIC), Isaac Newton 8 (PTM) 28760 Tres Cantos, Madrid, Spain
}

(Received 23 January 2006; published 2 May 2006)

\begin{abstract}
A theoretical formalism that allows analysis of the magneto-optical response of nanocorrugated ferromagnetic surfaces is presented and its validity checked with measurements in expressly fabricated structures. The formalism uses conventional scattering theory to find the expressions that account for the power scattered per unit area, and finds that for particular light-scattering directions and incidence polarizations the topographic and the magnetic contributions to the scattered light can be separated. By comparing theoretical and experimental results the magnetic state of the surfaces and its field evolution can be extracted.
\end{abstract}

DOI: 10.1103/PhysRevB.73.205402

PACS number(s): 78.67.-n, 61.46.-w, 72.20.My, 78.20.Ls

\section{INTRODUCTION}

Light scattering has been widely used as a method to characterize different types of rough surfaces. ${ }^{1,2}$ The intensity and polarization state of the scattered light depend not only on the topography of the surface but also on its optical properties. In the case of a ferromagnetic material the nonhomogeneous distribution of the magnetization in the surface produces an extra mechanism of light scattering. ${ }^{3}$ Moreover, the magnetic character of the material induces a magnetic field dependence of the scattered light, which for the case of periodic arrays of ferromagnetic elements such as gratings, tiles, or dots has been used to analyze their magnetic properties ${ }^{4-10}$ In this work these studies are extended to analyze the magnetic response of nanocorrugated surfaces. First, a theoretical formalism needed to treat these corrugated surfaces is presented, paying special attention to the transverse Kerr configuration geometry. Second, experimental results are obtained for periodically nanocorrugated Co surfaces, and compared with the theoretical predictions.

\section{THEORETICAL FORMALISM}

In a ferromagnetic material the dielectric tensor can be written as

$$
\left(\begin{array}{ccc}
\varepsilon_{x x} & -\hat{\varepsilon}_{x y} m_{z} & \hat{\varepsilon}_{x z} m_{y} \\
\hat{\varepsilon}_{x y} m_{z} & \varepsilon_{y y} & -\hat{\varepsilon}_{y z} m_{x} \\
-\hat{\varepsilon}_{x z} m_{y} & \hat{\varepsilon}_{y z} m_{z} & \varepsilon_{z z}
\end{array}\right),
$$

with $m_{l}, l=x, y, z$, the $l$ component of the normalized magnetization, defined as $m_{l}=\frac{M_{l}}{M_{\text {saturation }}} . M_{\text {saturation }}$ is the value of the magnetization at saturation and $M_{l}$ the $l$ component of the magnetization; $\hat{\varepsilon}_{i j}$ is a magneto-optical parameter, which depends on the nature of the material. For a material with cubic symmetry $\varepsilon_{x x}=\varepsilon_{y y}=\varepsilon_{z z}=\varepsilon$ and $\hat{\varepsilon}_{x y}=\hat{\varepsilon}_{y z}=\hat{\varepsilon}_{x z}=\varepsilon_{m o}$.

In those materials there are two different sources of light scattering. One is due to surface roughness or fluctuation in the refractive index. This source of scattering modifies the diagonal components of the dielectric tensor $\left(\Delta \varepsilon_{i i}, i=x, y, z\right)$. The other source of scattering is related to the fluctuations in the magneto-optical properties of the material and modifies the nondiagonal components of the dielectric tensor $\left(\Delta \varepsilon_{i j}, i\right.$ $\neq j ; i, j=x, y, z)$. The magneto-optical fluctuations can be due either to fluctuations in the chemical composition of the material (which modifies the magneto-optical parameter $\varepsilon_{m o}$ ) or to a nonhomogeneous distribution of the magnetization $m_{l}$, $l=x, y, z$. Therefore, the fluctuations of the dielectric tensor take in general the form

$$
\Delta \bar{\varepsilon}\left(\vec{r}^{\prime}\right)=\left(\begin{array}{ccc}
\Delta \varepsilon_{x x}\left(\vec{r}^{\prime}\right) & \Delta \varepsilon_{x y}\left(\vec{r}^{\prime}\right) & \Delta \varepsilon_{x z}\left(\vec{r}^{\prime}\right) \\
\Delta \varepsilon_{y x}\left(\vec{r}^{\prime}\right) & \Delta \varepsilon_{y y}\left(\vec{r}^{\prime}\right) & \Delta \varepsilon_{y z}\left(\vec{r}^{\prime}\right) \\
\Delta \varepsilon_{z x}\left(\vec{r}^{\prime}\right) & \Delta \varepsilon_{z y}\left(\vec{r}^{\prime}\right) & \Delta \varepsilon_{z z}\left(\vec{r}^{\prime}\right)
\end{array}\right),
$$

with $\Delta \varepsilon_{i j}\left(\vec{r}^{\prime}\right)=-\Delta \varepsilon_{j i}\left(\vec{r}^{\prime}\right) ; i \neq j$.

If only first-order scattering is considered, the electric field component of the scattered light induced by these optical and magneto-optical fluctuations can be obtained from the zero-order field $\left[\vec{E}^{0}(\vec{r})\right.$, i.e., the electric field solution of the problem without fluctuations] as follows:

$$
\vec{E}^{\text {scatt }}(\vec{r}) \propto \int d^{3} r^{\prime} \vec{G}\left(\vec{r}, \vec{r}^{\prime}\right) \Delta \bar{\varepsilon}\left(\vec{r}^{\prime}\right) \vec{E}^{0}\left(\vec{r}^{\prime}\right),
$$

where $\vec{G}\left(\vec{r}, \vec{r}^{\prime}\right)$ is a matrix Green function, satisfying the corresponding equation; see Refs. 11-13,

$$
\begin{aligned}
& \sum_{j}\left(\frac{2 \pi}{\lambda} \varepsilon_{i j} \delta_{i j}-\left[1+\frac{2 \pi}{\lambda} \varepsilon_{i j}\left(1-\delta_{i j}\right)\right] \frac{\partial^{2}}{\partial r_{i} \partial r_{j}}+\delta_{i j} \nabla^{2}\right) G_{j k}\left(\bar{r}, \bar{r}^{\prime}\right) \\
& \quad=4 \pi \delta_{i k} \delta\left(\bar{r}-\bar{r}^{\prime}\right) .
\end{aligned}
$$

After some algebra, the scattered electric and magnetic components of the electromagnetic field in the plane of incidence $\left(k_{y}=0\right)$ can be obtained and are proportional to

$$
\begin{gathered}
\vec{E}^{\text {scatt }} \propto \int d k_{x}\left[\left(\hat{x} k_{z}-\hat{z} k_{x}\right) M\left(k_{x}\right)+(2 \pi / \lambda) \hat{y} N\left(k_{x}\right)\right] e^{i\left(k_{x} x+k_{z} z\right)}, \\
\vec{H}^{\text {scatt }} \propto \int d k_{x}\left[\left(\hat{x} k_{z}-\hat{z} k_{x}\right) N\left(k_{x}\right)-\left(2 \pi \varepsilon_{0} / \lambda\right) \hat{y} M\left(k_{x}\right)\right] e^{i\left(k_{x} x+k_{z} z\right)},
\end{gathered}
$$

where $k_{x}=\frac{2 \pi}{\lambda} \sin \theta, k_{z}=\frac{2 \pi}{\lambda} \sqrt{\varepsilon_{0}-\sin ^{2} \theta}, \varepsilon_{0}$ is the diagonal dielectric constant of the incident medium, $\theta$ the scattering angle, and $\lambda$ the wavelength, 


$$
\begin{aligned}
M\left(k_{x}\right)= & \left(2 \pi / \lambda k_{z}\right) \int d z^{\prime}\left(\Delta \varepsilon\left(\widetilde{g}_{x x} E_{x}^{0}+\widetilde{g}_{x y} E_{y}^{0}+\widetilde{g}_{x z} E_{z}^{0}\right)\right. \\
& +\Delta \varepsilon_{x y}\left(\widetilde{g}_{x x} E_{y}^{0}-\widetilde{g}_{x y} E_{x}^{0}\right)+\Delta \varepsilon_{x z}\left(\widetilde{g}_{x x} E_{z}^{0}-\widetilde{g}_{x z} E_{x}^{0}\right) \\
& \left.+\Delta \varepsilon_{y z}\left(\widetilde{g}_{x y} E_{z}^{0}-\widetilde{g}_{x z} E_{y}^{0}\right)\right)
\end{aligned}
$$

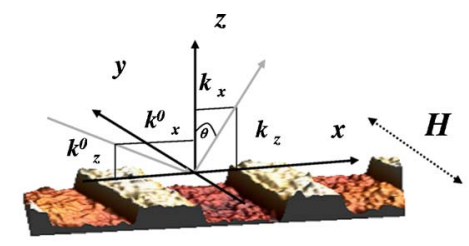

and

$$
\begin{aligned}
N\left(k_{x}\right)= & \int d z^{\prime}\left(\Delta \varepsilon\left(\widetilde{g}_{y x} E_{x}^{0}+\widetilde{g}_{y y} E_{y}^{0}+\widetilde{g}_{y z} E_{z}^{0}\right)+\Delta \varepsilon_{x y}\left(\widetilde{g}_{y x} E_{y}^{0}\right.\right. \\
& \left.-\widetilde{g}_{y y} E_{x}^{0}\right)+\Delta \varepsilon_{x z}\left(\widetilde{g}_{y x} E_{z}^{0}-\widetilde{g}_{y z} E_{x}^{0}\right)+\Delta \varepsilon_{y z}\left(\widetilde{g}_{y y} E_{z}^{0}\right. \\
& \left.\left.-\widetilde{g}_{y z} E_{y}^{0}\right)\right) .
\end{aligned}
$$

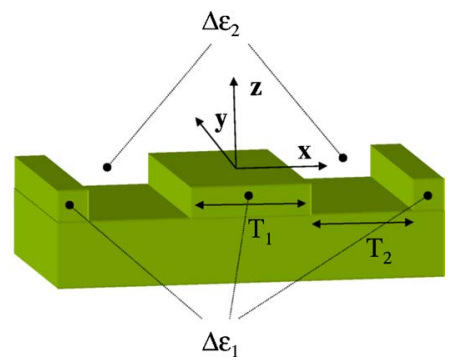

$\Delta \varepsilon_{\alpha \beta}$ are the Fourier-transformed component $\left(\Delta \varepsilon_{\alpha \beta}\left(k_{x}^{0}\right.\right.$ $\left.\left.-k_{x}, z^{\prime}\right)\right)$ of $\Delta \varepsilon_{\alpha \beta}\left(\bar{r}^{\prime}\right)$. The $\widetilde{g}_{i j}$ are functions obtained from the Fourier-transformed component $g_{i j}\left(z, z^{\prime}\right)$ (see Appendix A) of $\vec{G}\left(\vec{r}, \vec{r}^{\prime}\right):\left(g_{i j}\left(z, z^{\prime}\right)=\widetilde{g}_{i j} e^{i z_{z}}\right)$, and $E_{\beta}^{0}$ is the $\beta$ component of the zero-order electric field transmitted inside the reflecting medium $\left(E_{\beta}^{0}=E_{\beta 1}^{0} e^{i k_{z 1}^{0} z^{\prime}}\right.$ with $k_{z 1}^{0}=-\frac{2 \pi}{\lambda} \sqrt{\varepsilon-\sin ^{2} \theta_{0}}$, where $\varepsilon$ is the diagonal dielectric constant of the reflecting medium and $\theta_{0}$ the incident angle). It has been assumed $\Delta \varepsilon_{x x}\left(k_{x}^{0}-k_{x}, z^{\prime}\right)$ $=\Delta \varepsilon_{y y}\left(k_{x}^{0}-k_{x}, z^{\prime}\right)=\Delta \varepsilon_{z z}\left(k_{x}^{0}-k_{x}, z^{\prime}\right)=\Delta \varepsilon\left(k_{x}^{0}-k_{x}, z^{\prime}\right)$ (for simplicity their $k_{0 x}-k_{x}, z^{\prime}$ dependence will not be written in the expressions.

Therefore, the power scattered per unit area along the direction defined by the unit vector $\left(\frac{k_{x}}{K}, 0, \frac{k_{z}}{K}\right), K=\sqrt{k_{x}^{2}+k_{z}^{2}}$, is proportional to

$$
\left[\left\langle N N^{*}\right\rangle+\left\langle M M^{*}\right\rangle\right]
$$

The \langle\rangle stands for spatial average. The $M$ term represents the $p$-polarized scattered light and the $N$ term the $s$-polarized scattered light.

For an arbitrary direction of the magnetization all terms in the $N$ and $M$ factors contribute to the scattering, but some simplifications can be done for especial cases. In particular, if the magnetization is aligned along the $y$ axis (transversal configuration, see Fig. 1), the dielectric tensor of the homogeneous system can be written as

$$
\left(\begin{array}{ccc}
\varepsilon & 0 & \varepsilon_{m o} m_{y} \\
0 & \varepsilon & 0 \\
-\varepsilon_{m o} m_{y} & 0 & \varepsilon
\end{array}\right) .
$$

Such dielectric tensor does not couple the $P$ and $S$ components of the electric field and, therefore, an incident beam $p$ polarized ( $s$ polarized) will remain $p(s)$ polarized in the material. Furthermore, for this type of dielectric tensor some of the $g_{i j}$ functions are zero (see Appendix A).

If the incident light is $S$ polarized, the $M$ and $N$ terms take the form

$$
\left.M\left(k_{x}\right)=\left(2 \pi / \lambda k_{z}\right) \int d z^{\prime}\left(\left(\Delta \varepsilon_{x y} \widetilde{g}_{x x}-\Delta \varepsilon_{y z} \widetilde{g}_{x z}\right) E_{y}^{0}\right)\right)
$$

and

FIG. 1. (Color online) (A) Transversal MOKE geometry shown on an AFM image of the nanocorrugation: incident and scattered light $p$ polarized, external magnetic field applied perpendicular to the incidence plane and along the stripes' long axis (the diffraction spots appear at the incidence plane). (B) Sample structure with the geometrical and optical parameters used in the theory

$$
N\left(k_{x}\right)=\int d z^{\prime}\left(\Delta \varepsilon \widetilde{g}_{y y} E_{y}^{0}\right) .
$$

Notice that in this configuration the $s$ component of the scattered light $\left(I_{s \rightarrow s}^{\text {scatt }} \propto\left\langle N N^{*}\right\rangle\right)$ is only originated by nonmagnetic dielectric fluctuations $(\Delta \varepsilon)$. On the other hand, the $p$ component of the scattered light $\left(I_{s \rightarrow p}^{s c a t t} \propto\left\langle M M^{*}\right\rangle\right)$ is solely originated by magneto-optical fluctuations $\left(\Delta \varepsilon_{x y}, \Delta \varepsilon_{y z}\right)$.

If the incident light is $P$ polarized, the $M$ and $N$ terms can be written as

$$
\begin{aligned}
M\left(k_{x}\right)= & \left(2 \pi / \lambda k_{z}\right) \int d z^{\prime}\left(\Delta \varepsilon\left(\widetilde{g}_{x x} E_{x}^{0}+\widetilde{g}_{x z} E_{z}^{0}\right)\right. \\
& \left.+\Delta \varepsilon_{x z}\left(\widetilde{g}_{x x} E_{z}^{0}-\widetilde{g}_{x z} E_{x}^{0}\right)\right)
\end{aligned}
$$

and

$$
N\left(k_{x}\right)=\int d z^{\prime}\left(\left(\Delta \varepsilon_{y z} E_{z}^{0}-\Delta \varepsilon_{x y} E_{x}^{0}\right) \widetilde{g}_{y y}\right) .
$$

In this case, the $s$ component of the scattered light $\left(I_{p \rightarrow s}^{\text {scatt }}\right.$ $\left.\propto\left\langle N N^{*}\right\rangle\right)$ has only magneto-optical sources of scattering $\left(\Delta \varepsilon_{x y}, \Delta \varepsilon_{y z}\right)$. On the other hand, the $p$ component of the scattered light $\left(M M^{*}\right.$ term) has two types of scattering sources, one which depends on the nonmagnetic dielectric fluctuations $(\Delta \varepsilon)$ and the other which depends on the magnetooptical fluctuations $\left(\Delta \varepsilon_{x z}\right)$. In a homogeneous material $\left(\varepsilon_{m o}\right.$ $=$ const.), the magneto-optical fluctuations can be the result of a nonhomogeneous distribution of the magnetization, like one due to a magnetic domain structure present in the sample $\left\{\Delta \varepsilon_{x z}=\varepsilon_{m o}\left[m_{y}(\vec{r})-\widetilde{m}_{y}\right]\right.$, with $\widetilde{m}_{y}$ the mean value of the $y$ component of the normalized magnetization\}. However, at magnetic saturation, the fluctuations in $\Delta \varepsilon_{x z}$ arise solely from the fluctuations in the magneto-optical properties of the surface. If the fluctuations in the dielectric tensor are localized in a 
very thin region, of thickness $h$, underneath the surface, the $p$ scattered light intensity is proportional to

$$
\begin{aligned}
I_{p \rightarrow p}^{\text {scatt }} \propto & {\left[\Delta \varepsilon^{\text {surface }}\left(k_{x}^{0}-k_{x}\right) h\right]^{2} \mid\left(\left(f_{x x} E_{x 1}^{0}+f_{x z} E_{z 1}^{0}\right)+Q_{s}\left(k_{x}^{0}-k_{x}\right)\right.} \\
& \left.\times\left(f_{x x} E_{z 1}^{0}-f_{x z} E_{x 1}^{0}\right)\right)\left.\right|^{2},
\end{aligned}
$$

where the $f_{i j}$ functions are defined in Appendix A, and $E_{x 1}^{0}$, $E_{z 1}^{0}$ are the $x$ and $z$ components of the zero-order transmitted electrical field, $\Delta \varepsilon^{\text {surface }}\left(k_{x}^{0}-k_{x}\right)$ is the Fourier transform component of the optical fluctuations in this surface region, $Q_{s}\left(k_{x}^{0}-k_{x}\right)$ is defined as $Q_{s}\left(k_{x}^{0}-k_{x}\right)=\frac{\Delta \varepsilon_{x z}^{\text {sufface }}\left(k_{x}^{0}-k_{x}\right)}{\Delta \varepsilon^{\text {sufface }}\left(k_{x}^{0}-k_{x}\right)}$, and is related to the magneto-optical fluctuations at the surface region.

Therefore, the magneto-optical scattering ratio, MOSR, is

$$
\operatorname{MOSR}=\frac{\Delta I_{p \rightarrow p}}{I_{p \rightarrow p}}=\frac{I_{p \rightarrow p}^{\text {scatt }}\left(m_{y}=1\right)-I_{p \rightarrow p}^{\text {scatt }}\left(m_{y}=-1\right)}{I_{p \rightarrow p}^{\text {scatt }}\left(m_{y}=1\right)+I_{p \rightarrow p}^{\text {scatt }}\left(m_{y}=-1\right)},
$$

and is obtained by measuring the intensity of the $p \rightarrow p$ polarized scattered light when the magnetization runs from saturation in one direction, $m_{y}=1$, to saturation in the opposite direction, $m_{y}=-1$, and can be written as

$$
\frac{\Delta I_{p \rightarrow p}}{I_{p \rightarrow p}}=\frac{S\left(k_{x}^{0}, k_{x}, \varepsilon, \varepsilon_{m o}, Q_{s}\right)-S\left(k_{x}^{0}, k_{x}, \varepsilon,-\varepsilon_{m o},-Q_{s}\right)}{S\left(k_{x}^{0}, k_{x}, \varepsilon, \varepsilon_{m o}, Q_{s}\right)+S\left(k_{x}^{0}, k_{x}, \varepsilon,-\varepsilon_{m o},-Q_{s}\right)},
$$

with $\quad S\left(k_{x}^{0}, k_{x}, \varepsilon^{b}, \varepsilon_{t}^{b}, Q_{s}\right)=\mid\left(\left(f_{x x} E_{x 1}^{0}+f_{x z} E_{z 1}^{0}\right)+Q_{s}\left(k_{x}^{0}-k_{x}\right)\right.$ $\left.\times\left(f_{x x} E_{z 1}^{0}-f_{x z} E_{x 1}^{0}\right)\right)\left.\right|^{2}$.

The MOSR is a function of the incident angle $\left(k_{x}^{0}\right)$, the scattering angle $\left(k_{x}\right)$, and depends only on the optical $(\varepsilon)$ and magneto-optical $\left(\varepsilon_{m o}\right)$ properties of the bulk, and on the magneto-optical properties of the surface $\left(\mathrm{Q}_{s}\right)$. Therefore, it is a fingerprint of the magnetic nature of the surface region. If the magneto-optical fluctuations, $\Delta \varepsilon_{x z}^{\text {surface }}\left(k_{x}^{0}-k_{x}\right)$, and the optical fluctuations, $\Delta \varepsilon^{\text {surface }}\left(k_{x}^{0}-k_{x}\right)$, of the surface have the same spatial distribution, $Q_{s}$ does not depend on $\left(k_{x}^{0}-k_{x}\right)$.

\section{RESULTS AND DISCUSSION}

One-dimensional corrugated surfaces have been fabricated using conventional "lift-off" techniques. On glass substrates continuous layers of $\mathrm{Co}$ or $\mathrm{Au}$ (thick enough to be opaque) were grown by triode sputtering. After that $\mathrm{Co}$ or $\mathrm{Cr}$ arrays of stripes, with a height of 5-7 nm, were fabricated on the top of the previous metallic layer by lift-off processing. The stripes have a width varies between 2 and $4 \mu \mathrm{m}$ and the separation between the stripes ranges between 4 and $8 \mu \mathrm{m}$. Three different types of nanocorrugated structures were fabricated. In the first type the Co stripes were deposited on top of a continuous Au layer. In the second type $\mathrm{Cr}$ stripes were deposited on top of a Co layer, and in the third type Co stripes were deposited on top of a Co layer. Therefore, the three type of structures obtained consist of (i) a magnetic surface on top of a nonmagnetic substrate (Co stripes on $\mathrm{Au}$, termed type I); (ii) a nonmagnetic surface on top of a magnetic substrate ( $\mathrm{Cr}$ stripes on $\mathrm{Co}$, type II); and finally (iii) a magnetic surface on top of a magnetic substrate (Co stripes on Co, type III). Note that due to the fabrication process a thin oxide layer will be located between the con-

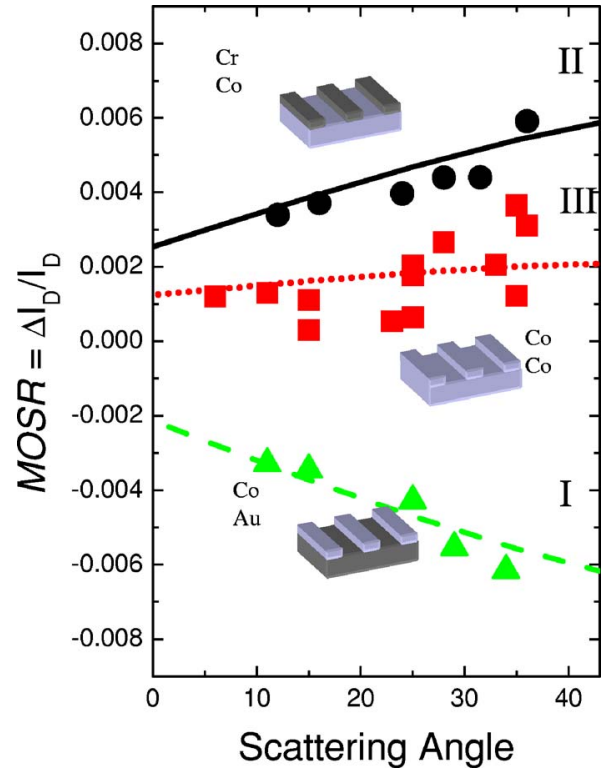

FIG. 2. (Color online) Magneto-optical scattering ratio as a function of the scattering angle for $20^{\circ}$ incidence angle. The lines are the theoretical results for (i) a magnetic surface on top of a nonmagnetic substrate (Co stripes on $\mathrm{Au}$, termed type I); (ii) a nonmagnetic surface on top of a magnetic substrate $(\mathrm{Cr}$ stripes on Co, type II); and finally (iii) a magnetic surface on top of a magnetic substrate (Co stripes on Co, type III). The solid symbols are experimental results for the fabricated structures.

tinuous layer of $\mathrm{Co}$ and the stripes. The Co grown on glass by triode sputtering develops a uniaxial anisotropy parallel to the plasma confining magnetic field, which for a thick enough layer has an anisotropy field of about 30 Oe.

Figure 2 presents the experimental magneto-optical scattering ratio as a function of the scattering angle for the three types of structure (I, II, and III) obtained by the abovementioned methods (solid symbols). The incident light angle is $20 \mathrm{deg}$ and its wavelength $638 \mathrm{~nm}$ (He-Ne laser). As observed, the experimental MOSR depends on the type of structure. Also, in Figure 2 the theoretical MOSR ratio is represented by lines. The solid line corresponds to a structure where the substrate is magnetic and the surface is nonmagnetic $\left(Q_{s}=0\right)$, which fits the data for structures of $\mathrm{Cr}$ stripes on Co, type II. The dotted line corresponds to a structure where both the substrate and the surface are magnetic (Co stripes on Co, type III), and the dashed line corresponds to a structure where the surface is magnetic and the substrate is nonmagnetic (Co stripes on Au, type I). The optical constant of $\mathrm{Co}$ and $\mathrm{Au}$ used to calculate the MOSR were obtained from Ref. 14, the magneto-optical constants of the continuous Co layer were obtained from experimental magnetooptical measurements performed in noncorrugated Co layers $\left(\varepsilon_{x y}=0.14+i .25\right)$, and the $Q_{s}$ of the Co stripes was obtained from a fit of the MOSR ratio for the Co stripes on $\mathrm{Au}\left(Q_{s}\right.$ $=-0.01 i)$. As observed, the theoretical curves reflect the different behavior of the three types of structures, providing information about the magnetic nature of the surface.

Figure 3 presents the reflected and diffracted MOKE loops for the three kinds of structures. The easy magnetiza- 


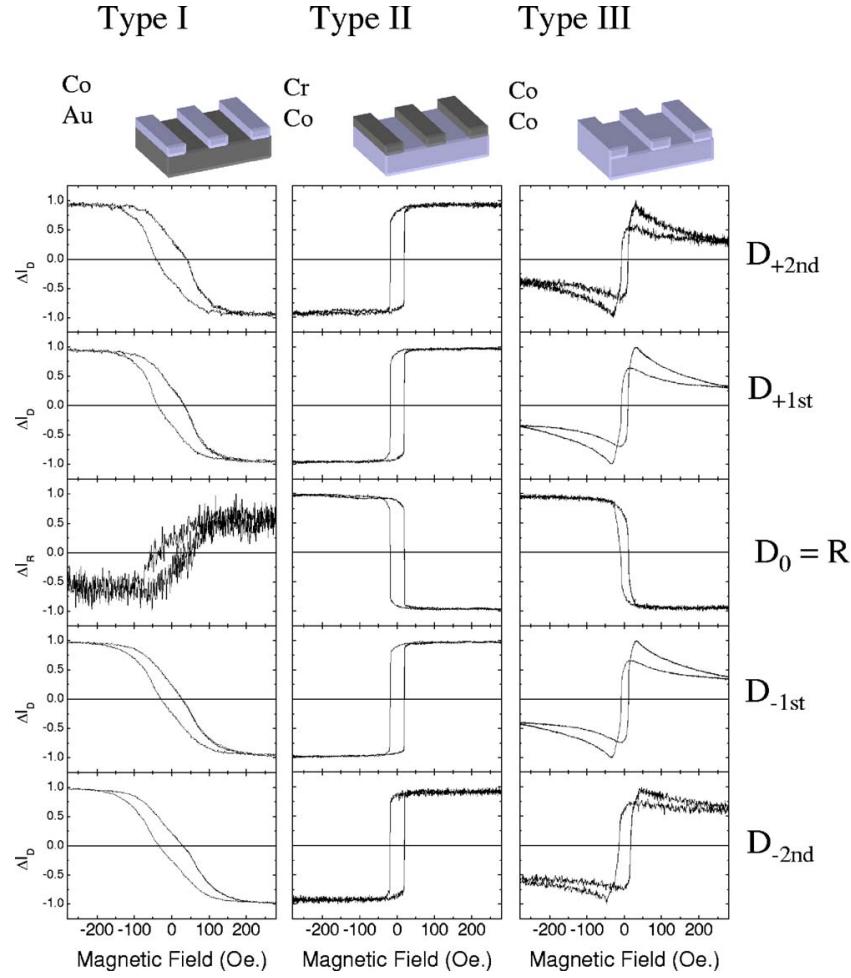

FIG. 3. (Color online) Transverse Kerr hysteresis loops for the direct (reflected) and the first and second diffracted spots, measured at $20^{\circ}$ incidence angle, for type I, type II, and type III structures. The easy magnetization axis of the Co layer is parallel to the stripes long axis.

tion axis of the sputtered Co was parallel to the stripes' long axis. Depending on the type of structure, the magnetic field dependence of the reflected and the diffracted MOKE intensities is different. In particular, the shapes of the different MOKE loops of the type I structure are very similar and reflect the magnetic field dependence of the stripe magnetization. For type II structures the continuous Co layer is the only magnetic material and therefore the magneto-optic component is that of the continuous Co layer. In these two structures the shape of the different reflected and diffracted loops is very similar. However, in type III structures the magnetic field dependence of the reflected and diffracted beams is quite different. For this structure, the thin oxide layer between the stripes and the continuous layer exchange decouples their magnetization. ${ }^{15}$ Therefore, the magnetic field dependence of the stripe and continuous magnetization are independent and should be very similar to the one obtained in type I and II, respectively. As seen in Figure 3, the magnetic field dependence of the reflected beam shows the evolution of the magnetization of the continuous Co layer, whereas the magnetic field dependence of the diffracted beams intensities, which are generated by the nanocorrugated surface, reflect the evolution of both continuous layer and stripe magnetizations.

In fact, if the only source of light scattering is the nanocorrugated surface the magnetic field dependence of the diffracted light is proportional to

$$
\begin{aligned}
S\left(k_{x}^{0}, k_{x}, \varepsilon, \varepsilon_{m o} m_{y}^{b}, Q_{s}\right)= & \mid\left(\left(f_{x x} E_{x 1}^{0}+f_{x z} E_{z 1}^{0}\right)\right. \\
& \left.+Q_{s}\left(f_{x x} E_{z 1}^{0}-f_{x z} E_{x 1}^{0}\right)\right)\left.\right|^{2},
\end{aligned}
$$

and, we can use this expression to calculate the theoretical magnetic field dependence of the diffracted light intensity. For that purpose we use for the magnetic field dependence of the $y$ component of the continuous layer magnetization $\left(m_{y}^{b}\right)$ the magnetic field dependence of the reflected beam, and we assume that in the stripes the magnetization is homogeneous. In that case $Q_{s}(k)=\widetilde{Q}_{s} \widetilde{m}_{y}^{\text {stripe }}$, with $\widetilde{m}_{y}^{\text {stripe }}$ the mean value of the $y$ component of the normalized stripe magnetization, whose dependence on the magnetic field is similar to that given by the type I loops, and $\widetilde{Q}_{s}=-0.01 i$ for our fabricated Co stripes

As an example, Fig. 4 presents the experimental [Fig. 4(a)] and the simulated magnetic field dependence [Fig. 4(b)] of the intensity of the -1 diffracted spot for two type III samples where the easy axis of the continuous Co layer is in one case parallel to the stripe long axis (sample A) and in the other perpendicular to that axis (sample B). The simulated Kerr loops of the diffracted spots [Fig. 4(b)] were obtained as explained in the previous paragraph: the magnetic field dependence of continuous layer magnetization $m_{y}^{b}$ [Fig. 4(d)] is assumed to be the one given by the experimental reflected Kerr loops of the structures [upper part of Fig. 4(a)], and we use for the magnetic field dependence $\widetilde{m}_{y}^{\text {stripe }}$ of the two samples [Fig. 4(c)] the same magnetic field dependence as that of type I loops.

As observed, the shape of the experimental [lower part of Fig. 4(a)] and simulated Kerr Loops [Fig. 4(b)] are very similar, which confirms the validity of the model and strongly supports the suggestion that in type III structures the stripes and the continuous Co layer are exchange decoupled.

\section{CONCLUSIONS}

Summarizing, a formalism that accounts for the magnetooptical response of a periodic magnetic nanocorrugation is presented. The theory uses conventional scattering theory to find the expressions that account for the power scattered per unit area, and finds that for particular light-scattering directions and incidence polarizations the topographic and the magnetic contributions to the scattered light can be separated. This fact is used to find the magnetic state of the nanocorrugated surface. Furthermore, with reasonable assumptions using the reflected and diffracted MO components, the detailed magnetic behavior (hysteresis loop) of the surface can be extracted and compared with the bulk. This opens interesting possibilities for the characterization of interfacial magnetism and in particular buried interfaces which cannot be obtained using other methods.

\section{ACKNOWLEDGMENT}

This work was supported by the Ministerio de Ciencia y Tecnología, Project MAT2002-04484-C03-01. 

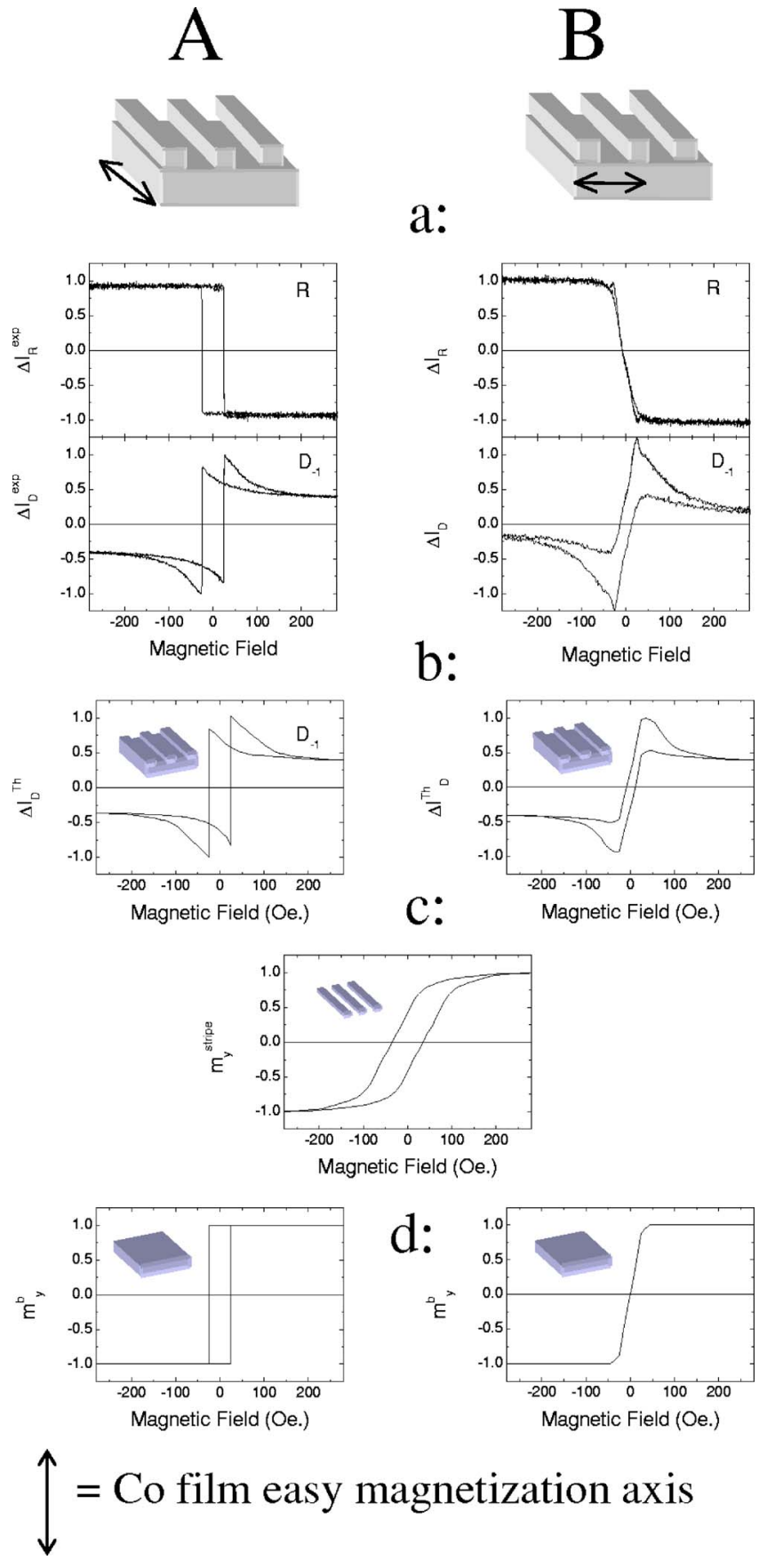

FIG. 4. (Color online) Experimental and simulated hysteresis Kerr loops for the reflected and first-order diffracted spots for two different orientations of the easy axis of the Co film with respect of the applied field. (A) easy axis parallel to the stripe long axis. (B) easy axis perpendicular to the stripe long axis. (a) Experimental reflected Kerr loops $(R)$, and first-order diffracted Kerr loops $\left(D_{-1}\right)$. (b) Simulated first-order diffracted Kerr loops. (c) Magnetic field dependence of the y component of the stripe magnetization.

\section{APPENDIX A}

If we are interested only in the scattered light in the plane of incidence $\left(k_{y}=0\right)$, the functions $g_{i j}$ satisfy the following equations:

$$
\left(\begin{array}{ccc}
\widetilde{\varepsilon}_{0}+\frac{d}{d^{2} z} & 0 & -i k_{x} \frac{d}{d z} \\
0 & \widetilde{\varepsilon}_{0}-k_{x}^{2}+\frac{d}{d^{2} z} & 0 \\
-i k_{x} \frac{d}{d z} & 0 & \widetilde{\varepsilon}_{0}-k_{x}^{2}
\end{array}\right)\left(\begin{array}{ccc}
g_{x x} & g_{x y} & g_{x z} \\
g_{y x} & g_{y y} & g_{y z} \\
g_{z x} & g_{z y} & g_{z z}
\end{array}\right)
$$

for $z>0$ (incidence medium), and for $z<0$ (magnetic material) in the transversal configuration

$$
\left(\begin{array}{ccc}
\widetilde{\varepsilon}+\frac{d}{d^{2} z} & 0 & \widetilde{\varepsilon}_{t}-i k_{x} \frac{d}{d z} \\
0 & \widetilde{\varepsilon}-k_{x}^{2}+\frac{d}{d^{2} z} & 0 \\
-\widetilde{\varepsilon}_{t}-i k_{x} \frac{d}{d z} & 0 & \widetilde{\varepsilon}-k_{x}^{2}
\end{array}\right)\left(\begin{array}{lll}
g_{x x} & g_{x y} & g_{x z} \\
g_{y x} & g_{y y} & g_{y z} \\
g_{z x} & g_{z y} & g_{z z}
\end{array}\right)
$$

where $k_{x}=\frac{2 \pi}{\lambda} \sin \theta, k_{z}=\frac{2 \pi}{\lambda} \sqrt{\varepsilon_{0}-\sin ^{2} \theta}, k_{z 1}=-\frac{2 \pi}{\lambda} \sqrt{\varepsilon-\sin ^{2} \theta}, \widetilde{\varepsilon}$ $=\varepsilon\left(\frac{2 \pi}{\lambda}\right)^{2}, \widetilde{\varepsilon}_{0}=\varepsilon_{0}\left(\frac{2 \pi}{\lambda}\right)^{2}$, and $\widetilde{\varepsilon}_{t}=\varepsilon_{m o} m_{y}\left(\frac{2 \pi}{\lambda}\right)^{2}$.

The solutions of these equations satisfy some conditions: for $z= \pm \infty$ (waves with exponential decay) and for the interface between the two media at $z=0$. In this case the boundary conditions are obtained from the continuity of the electromagnetic fields and at $z=0$ they give rise to the continuity of the following quantities:

(i) $g_{x x}, g_{x y}, g_{x z}$;

(ii) $g_{y x}, g_{y y}, g_{y z}$;

(iii) $\varepsilon_{z x} g_{x x}+\varepsilon_{z y} g_{y x}+\varepsilon_{z z} g_{z x}$;

(iv) $\varepsilon_{z x} g_{x y}+\varepsilon_{z y} g_{y y}+\varepsilon_{z z} g_{z y}$;

(v) $\varepsilon_{z x} g_{x z}+\varepsilon_{z y} g_{y z}+\varepsilon_{z z} g_{z z}$.

In particular $g_{x y}, g_{y x}, g_{y z}, g_{z y}$ are zero and

$$
\begin{gathered}
g_{x x}=\frac{\lambda^{2}}{\pi} i \frac{k_{z 1}^{2} k_{z}}{\left(k_{z} k_{z 1} \varepsilon-\varepsilon_{0} k_{z 1}^{2}\right)-\varepsilon_{m o} m_{y} k_{x} k_{z}} e^{i k_{z 1} z^{\prime}} e^{i k_{z} z}, \\
g_{z x}=-\frac{\lambda^{2}}{\pi} i \frac{k_{z 1}^{2} k_{x}}{\left(k_{z} k_{z 1} \varepsilon-\varepsilon_{0} k_{z 1}^{2}\right)-\varepsilon_{m o} m_{y} k_{x} k_{z}} e^{i k_{z 1} z^{\prime}} e^{i k_{z} z}, \\
g_{y y}=\frac{4 \pi i}{k_{z 1}-k_{z}} e^{i k_{z 1} z^{\prime}} e^{i k_{z} z} ; \\
g_{x z}=\frac{\lambda^{2}}{\pi} i \frac{k_{z}\left(k_{x} k_{z 1}-\varepsilon_{m o} m_{y} \frac{4 \pi^{2}}{\lambda^{2}}\right)}{\left(k_{z} k_{z 1} \varepsilon-\varepsilon_{0} k_{z 1}^{2}-\varepsilon_{m o} m_{y} k_{x} k_{z}\right)} e^{i k_{z 1} z^{\prime}} e^{i k_{z} z}, \\
g_{z z}=\frac{\lambda^{2}}{\pi} i \frac{k_{x}\left(k_{x} k_{z 1}-\varepsilon_{m o} m_{y} \frac{4 \pi^{2}}{\lambda^{2}}\right)}{\left(k_{z} k_{z 1} \varepsilon-\varepsilon_{0} k_{z 1}^{2}-\varepsilon_{m o} m_{y} k_{x} k_{z}\right)} e^{i k_{z 1} z^{\prime}} e^{i k_{z} z},
\end{gathered}
$$

when $z>0$ (air) and $z^{\prime}<0$ (scattering sources).

$$
f_{x x}=\frac{\lambda^{2}}{\pi} i \frac{k_{z 1}^{2} k_{z}}{\left(k_{z} k_{z 1} \varepsilon-\varepsilon_{0} k_{z 1}^{2}\right)-\varepsilon_{m o} m_{y} k_{x} k_{z}},
$$




$$
f_{x z}=\frac{\lambda^{2}}{\pi} i \frac{k_{z}\left(k_{x} k_{z 1}-\varepsilon_{m o} m_{y} \frac{4 \pi^{2}}{\lambda^{2}}\right)}{\left(k_{z} k_{z 1} \varepsilon-\varepsilon_{0} k_{z 1}^{2}-\varepsilon_{m o} m_{y} k_{x} k_{z}\right)} .
$$

\section{APPENDIX B}

Let us consider a periodic one-dimensional structure, like the nanograting shown in Fig. 1(b), consisting of a periodic stripe array located on top of a thick layer. We denote the height of the stripe $h$ ( $h$ is very small), the width $T_{1}$, the interstripe distance $T_{2}$, and the period $T=T_{1}+T_{2}$. We will assume that the stripes have a dielectric constant and a magneto-optical coefficient different from that of the bulk material. Inside the stripes $\varepsilon=\varepsilon^{\text {stripe }}$ and $\varepsilon_{x z}=\varepsilon_{m o}^{\text {stripe }} m_{y}^{\text {stripe }}$, with $\varepsilon_{m o}^{\text {stripe }}$ the magneto-optical coefficient of the stripe material and $m_{y}^{\text {stripe }}$ the normalized $y$ component of the stripe magnetization, which may have a nonhomogeneous distribution inside the stripe. In the interstripe region of the nanograting $\varepsilon=\varepsilon_{0}$ and $\varepsilon_{x z}=0$.

Therefore, if we call $k=k_{x}^{0}-k_{x}$ the Fourier transform component of the optical fluctuations $\left[\Delta \varepsilon^{\text {surface }}(k)\right.$ $\left.=\int_{-\infty}^{+\infty} \Delta \varepsilon^{\text {surface }}(x) e^{i k x} d x\right]$ can be expressed as

$$
\begin{aligned}
\Delta \varepsilon^{\text {surface }}(k)= & (2 / k)\left(\Delta \varepsilon_{1} \sin \left(\frac{k T_{1}}{2}\right)+e^{i(k T / 2)} \Delta \varepsilon_{2} \sin \left(\frac{k T_{2}}{2}\right)\right) \\
& \times\left(\sum_{n=-\infty}^{n=\infty} e^{i n k T}\right)
\end{aligned}
$$

with $\Delta \varepsilon^{1}=\varepsilon^{\text {bulk }}-\varepsilon^{\text {stripe }}$ and $\Delta \varepsilon_{2}=\varepsilon^{\text {bulk }}-\varepsilon_{0}$.

Taking into account that $\sum_{n=-\infty}^{n=\infty} e^{i n k T}=\frac{2 \pi}{T} \sum_{n=-\infty}^{n=\infty} \delta\left(k-\frac{n 2 \pi}{T}\right)$, which means that $\Delta \varepsilon^{\text {surface }}(k)$ is 0 for every $k$ except for $k$ $=\frac{2 n \pi}{T}, n=0, \pm 1, \pm 2, \pm 3$, the expression (B1) can be simplified to

$$
\begin{aligned}
\Delta \varepsilon^{\text {surface }}(k)= & (4 \pi / k T)\left(\left(\Delta \varepsilon_{2}-\Delta \varepsilon_{1}\right) \cos \left(\frac{k T}{2}\right) \sin \left(\frac{k T_{2}}{2}\right)\right) \\
& \times\left(\sum_{n=-\infty}^{n=\infty} \delta\left(k-\frac{n 2 \pi}{T}\right)\right)
\end{aligned}
$$

or

$$
\begin{aligned}
\Delta \varepsilon^{\text {surface }}(k)= & (2 / n)\left(\left(\varepsilon^{\text {stripe }}-\varepsilon_{0}\right) \cos (n \pi) \sin \left(\frac{n \pi T_{2}}{T}\right)\right) \\
& \times \delta\left(k-\frac{n 2 \pi}{T}\right) ; n= \pm 1, \pm 2, \ldots,
\end{aligned}
$$

whereas for the magneto-optical fluctuation

$$
\begin{aligned}
\Delta \varepsilon_{x z}^{\text {surface }}(k)= & \left((2 / n) \varepsilon_{m o}^{\text {stripe }} \tilde{m}_{y}^{\text {stripe }} \cos (n \pi) \sin \left(\frac{n \pi T_{2}}{T}\right)\right. \\
& \left.+(2 \pi / T) \varepsilon_{m o}^{\text {stripe }} m_{y}^{\text {stripe }}(k)\right) \delta\left(k-\frac{n 2 \pi}{T}\right) \\
& n= \pm 1, \pm 2, \ldots,
\end{aligned}
$$

with $m_{y}^{\text {stripe }}(k)=\int_{T_{-1 / 2}}^{T_{1 / 2}}\left(m_{y}^{\text {stripe }}(x)-\tilde{m}_{y}^{\text {stripe }}\right) e^{i k x} d x, \tilde{m}_{y}^{\text {stripe }}$ being the mean value of the magnetization inside the stripe. Therefore, $Q_{s}(k)=\frac{\Delta \varepsilon_{x z}^{\text {surface }}(k)}{\Delta \varepsilon^{\text {surface }}(k)}$.

$$
\begin{gathered}
Q_{s}(k)=\hat{Q}_{s}\left(\tilde{m}_{y}^{\text {stripe }}+\frac{n \pi}{T \cos (n \pi) \sin \left(\frac{n \pi T_{2}}{T}\right)} m_{y}^{\text {stripe }}(k)\right) ; \\
k=\frac{2 \pi n}{T} ; n= \pm 1, \pm 2, \ldots,
\end{gathered}
$$

with $\hat{Q}_{s}=\frac{\varepsilon_{m o}^{\text {stripe }}}{\varepsilon^{\text {stripe }}-\varepsilon_{0}}$.

If the stripe is homogeneous magnetized $m_{y}^{\text {stripe }}(k)=0$ and $Q_{s}(k)=\widetilde{Q}_{s} \tilde{m}_{y}^{\text {stripe }}$.
*Email address: gaspar@imm.cnm.csic.es

${ }^{1}$ M. Bennet and L. Mattson, Introduction to Surface Roughness and Scattering (Optical Society of America, Washington, DC, 1999).

${ }^{2}$ J. C. Stover, Optical Scattering: Measurement and Analysis (SPIE, Bellingham, WA, 1995).

${ }^{3}$ M. U. Gonzalez, G. Armelles, C. Martinez Boubeta, and A. Cebollada, Appl. Phys. Lett. 82, 421 (2002).

${ }^{4}$ T. Schmitte, K. Westerholt, and H. Zabel, J. Appl. Phys. 92, 4524 (2002).

${ }^{5}$ Andreas Westphalen, Till Schmitte, Kurt Westerholt, and Hartmut Zabel, J. Appl. Phys. 97, 073909 (2005).

${ }^{6}$ Y. Pagani, D. Van Labeke, B. Guizal, A. Vial, F. Baida, Opt. Commun. 209, 237 (2002).

${ }^{7}$ P. Garcia-Mochales, J. L. Costa-Krämer, G. Armelles, F. Briones, D. Jaque, J. Martin, and Vicent, Appl. Phys. Lett. 81, 3206 (2002)
${ }^{8}$ M. Grimsditch and P. Vavassori, J. Phys.: Condens. Matter 16, R275 (2004).

${ }^{9}$ O. Geoffroy, D. Givord, Y. Otani, B. Pannetier, A. D. Santos, M. Schlenker, and Y. Souche, J. Magn. Magn. Mater., 121, 516 (1993).

${ }^{10}$ M. Grimsditch, P. Vavassori, V. Novosad, V. Metlushko, H. Shima, Y. Otani, and K. Fukamichi, Phys. Rev. B 65, 172419 (2002).

${ }^{11}$ A. A. Maradudin and D. L. Mills, Phys. Rev. B 11, 1392 (1975).

${ }^{12}$ J. M. Elson, Phys. Rev. B 30, 5460 (1984).

${ }^{13}$ D. L. Mills, A. A. Maradudin and E. Burstein, Ann. Phys. 56, 504 (1970).

${ }^{14}$ J. H. Weaver, C. Krafka, D. W. Lynch, E. E. Kock, in Physics Data: Optical Properties of Metals (Fachinformationszentrum, Karlsruhe, 1981).

${ }^{15}$ J. L. Costa-Krämer, C. Guerrero, S. Muelle, P. García-Mochales, and F. Briones, Nanotechnology 14, 239 (2003). 